\title{
Work distribution for a particle moving in an optical trap and non-Markovian bath ${ }^{\dagger}$
}

\author{
ALOK SAMANTA, K SRINIVASU and SWAPAN K GHOSH* \\ Theoretical Chemistry Section, Bhabha Atomic Research Centre, Mumbai 400085 \\ e-mail: skghosh $a$ barc.gov.in
}

\begin{abstract}
We propose a simple approach to derive an exact analytical expression of work distribution for a system consisting of a colloidal particle trapped in an optical harmonic potential well, which is being pulled at a constant velocity through a solution represented by a non-Markovian bath. The thermal environment is represented by a bath composed of an infinite set of harmonic oscillators, and a model Hamiltonian for the trapped colloidal particle is constructed by representing the interaction with the bath via linear dissipative mechanism. We have studied the effects of pulling time, pulling speed, and the adiabatic limit. It is also observed that only at long time the total work is completely converted into dissipative work.
\end{abstract}

Keywords. Jarzynski theorem; non-Markovian bath; optical trap.

\section{Introduction}

The concept of free energy has been of central importance in thermodynamics and statistical mechanics. The laws of thermodynamics, first formulated in the early nineteenth century, indicate that the increase in Gibbs free energy $\Delta F$ in a process and the mean work $\langle W\rangle$ needed to result into that increase are related by $\Delta F \leq\langle W\rangle$. The equality holds when the process is carried out reversibly and the inequality holds otherwise. The scenario changed in 1997, when Jarzynski ${ }^{1}$ derived an equality relating the free energy difference corresponding to two states of a system along a reaction coordinate, to the work done for irreversibly switching the system between the two states, as given by

$$
\exp \left(-\frac{\Delta F}{k_{B} T}\right)=\int \exp \left(-\frac{W}{k_{B} T}\right) P(W) \mathrm{d} W
$$

where $P(W)$ is the non-equilibrium distribution of the work $W$, defined as

$$
P(W)=\left\langle\delta\left(W-\int_{0}^{t}\left(\frac{\partial H}{\partial \tau}\right) \mathrm{d} \tau\right)\right\rangle,
$$

\footnotetext{
${ }^{\dagger}$ Dedicated to the memory of the late Professor S K Rangarajan *For correspondence
}

with the angular bracket \langle\rangle denoting the average taken over different realizations and $H$ is the system Hamiltonian. This remarkable identity provides a practical tool for determining the equilibrium free energy difference from processes carried out arbitrarily far away from equilibrium. The theorem has been tested experimentally using bio-molecules manipulated mechanically, ${ }^{2}$ and also colloidal particle in time-dependent laser traps. ${ }^{3-6}$ Common to all colloidal experiments, so far, is the fact that these laser traps generate harmonic potential albeit with a time-dependent center or 'spring constant'. Consequently, often the interesting distributions are Gaussian even though for certain quantities, non-Gaussian distributions can occur. The optical trap experiment ${ }^{7}$ provides a route to obtain the work distribution function $P(W)$, and hence a theoretical approach ${ }^{8,9}$ to obtain the universal properties of various nonequilibrium distribution functions $P(W)$ and also their explicit forms are of obvious interest. In recent years, ${ }^{10,11}$ there have been a number of theoretical results for the non-equilibrium work distribution function pertaining to a system consisting of a particle pulled by a moving optical trap through a solution represented by a Markovian bath. However, it is well-known that the non-Markovian effect is very important in many situations such as at low temperature, high density and in the over damped region. Hence our objective in this work is to incorporate explicitly the non-Markovian effect into the pro- 
bability distribution $P(W)$. For this purpose, as an illustrative example, we consider a model bath consisting of an infinite set of harmonic oscillators. The rest of the paper is organized as follows. The theoretical formalism is presented in section 2, where we discuss the model system and derive an analytical expression for the non-equilibrium distribution for the work. In section 3, the results on the numerically calculated non-equilibrium work distribution for various physical parameters have been discussed. Finally, we offer a few concluding remarks in section 4 .

\section{Theory}

The system of interest is a colloidal particle trapped in an optical harmonic potential well with its center being dragged with a uniform speed through a thermal environment, as in the experiment of Wang et $a l .{ }^{4} \mathrm{We}$ consider the colloidal particle to be in a nonMarkovian bath, and the pulling to be done in onedimension along the coordinate $Q$ by a harmonic guiding potential $U(Q)$, with force constant $k_{o p}$, moving at a constant velocity $v$, i.e.

$$
U(Q)=\frac{1}{2} k_{o p}(Q-v t)^{2} .
$$

The particle is assumed to interact via linear dissipative mechanism with the thermal environment, which is represented by a bath composed of an infinite set $(N)$ of harmonic oscillators coupled linearly to the particle coordinate $Q$. The Hamiltonian $H$ of the system is given as

$$
\begin{aligned}
H= & \frac{P^{2}}{2 M}+\frac{1}{2} k_{o p}(Q-v t)^{2} \\
& +\sum_{j}^{N}\left[\frac{p_{j}^{2}}{2 m_{j}}+\frac{1}{2} m_{j} \omega_{j}^{2}\left(q_{j}-\frac{c_{j} Q}{m_{j} \omega_{j}^{2}}\right)^{2}\right],
\end{aligned}
$$

where $Q, P$ and $M$ respectively denote, the coordinate, momentum and mass of the colloidal particle. The quantities $\left\{q_{j}, p_{j}\right\}$ for $j=1,2 \ldots N$ are the coordinates and the momenta of the harmonic oscillators representing the solvent, $m_{j}$ and $\omega_{j}$ represent the mass and frequency of the $j^{\text {th }}$ oscillator and $c_{j}$ 's are the arbitrary coupling constants. The bath part of the above model Hamiltonian was first proposed by $Z_{\text {wanzig }}{ }^{12}$ and is widely used for the study of barrier crossing ${ }^{13}$ non-equilibrium solvation dynamics and escape of particle from a potential well, ${ }^{14}$ many ultrafast nonlinear techniques, ${ }^{15}$ Landau diamagnetism in a dissipative quantum system, ${ }^{16}$ etc. We now use the following transformation

$$
\bar{q}_{j}=\frac{m_{j} \omega_{j}^{2}}{c_{j}} q_{j}, \bar{p}_{j}=\frac{c_{j}}{m_{j} \omega_{j}^{2}} p_{j}, \bar{m}_{j}=\frac{c_{j}^{2}}{m_{j} \omega_{j}^{4}},
$$

to rewrite the Hamiltonian given by (4) as

$$
\begin{aligned}
H= & \frac{P^{2}}{2 M}+\frac{1}{2} k_{o p}(Q-v t)^{2} \\
& +\sum_{j}\left[\frac{\bar{p}_{j}^{2}}{2 \bar{m}_{j}}+\frac{1}{2} \bar{m}_{j} \omega_{j}^{2}\left(\bar{q}_{j}-Q\right)^{2}\right] .
\end{aligned}
$$

The work done by pulling the particle for a time $t$ through the solution is defined as

$$
\int_{0}^{t}\left(\frac{\partial H}{\partial \tau}\right) \mathrm{d} \tau=\frac{k_{o p} v^{2} t^{2}}{2}-k_{o p} v \int_{0}^{t} Q(\tau) \mathrm{d} \tau,
$$

which can be evaluated by solving for $Q(\tau)$ from the equation of motion derived below. The Hamilton's equations corresponding to the Hamiltonian of (6) lead to the equations of motion given by

$$
\frac{\mathrm{d} P}{\mathrm{~d} t}=-k_{o p}(Q-v t)+\sum_{j} \bar{m}_{j} \omega_{j}^{2}\left(\bar{q}_{j}-Q\right),
$$

and

$$
\frac{\mathrm{d} \bar{p}_{j}}{\mathrm{~d} t}=-\bar{m}_{j} \omega_{j}^{2}\left(\bar{q}_{j}-Q\right),
$$

where $P=M(\mathrm{~d} Q / \mathrm{d} t)$ and $p_{j}=m_{j}\left(\mathrm{~d} q_{j} / \mathrm{d} t\right)$.

Now, solving for $\bar{q}_{j}$ from (9) and substituting in (8), one obtains the Langevin type of equation

$$
M \frac{\mathrm{d}^{2} Q}{\mathrm{~d} t^{2}}=-k_{o p}(Q-v t)-\frac{1}{M} \int_{0}^{t} \xi(t-\tau) P(\tau) \mathrm{d} \tau+\theta(t),
$$

where

$$
\xi(t)=\sum_{j} \bar{m}_{j} \omega_{j}^{2} \cos \left(\omega_{j} t\right)
$$


and

$$
\begin{aligned}
\theta(t)= & \sum_{j} \sqrt{\bar{m}_{j}} \omega_{j} \\
& {\left[\sqrt{\bar{m}_{j}} \omega_{j}\left(\bar{q}_{j}-Q\right) \cos \omega_{j} t+\frac{\bar{p}_{j}}{\sqrt{\bar{m}_{j}}} \sin \omega_{j} t\right] . }
\end{aligned}
$$

Since it is convenient to express the second integral in (7) in terms of the inverse Laplace transform as

$$
\int_{0}^{t} Q(\tau) \mathrm{d} \tau=\mathcal{L}^{-1}\left(\frac{\tilde{Q}(s)}{s}\right),
$$

we propose to solve the differential equation (10) in Laplace space by first taking the Laplace transform

$$
\tilde{f}(s)=\int_{0}^{\infty} \mathrm{d} t e^{-s t} f(t)
$$

of both sides. After some algebraic manipulations, the final expression for the coordinate of the colloidal particle in Laplace space is given by

$$
\begin{aligned}
\tilde{Q}(s) & =\frac{P(0)}{\left[M\left(s^{2}+\frac{k_{o p}}{M}\right)+\xi(s) s\right]} \\
& +\frac{Q(0)[M s+\xi(s)]}{\left[M\left(s^{2}+\frac{k_{o p}}{M}\right)+\xi(s) s\right]} \\
& +\frac{s^{2}\left[M\left(s^{2}+\frac{k_{o p}}{M}\right)+\xi(s) s\right]}{\theta(s)}, \\
& +\frac{\left.k^{2} v\left(s^{2}+\frac{k_{o p}}{M}\right)+\xi(s) s\right]}{[M}
\end{aligned}
$$

where $Q(0)$ and $P(0)$ respectively represent the initial position and the conjugate momentum of the colloidal particle. Substituting this expression of $\tilde{Q}(s)$ in (13), and combining with (7), one obtains the expression for the work given by

$$
\int_{0}^{t}\left(\frac{\partial H}{\partial \tau}\right) \mathrm{d} \tau=\frac{k_{o p} v^{2} t^{2}}{2}-k_{o p} v
$$

$$
\begin{aligned}
& \left\{P(0) f_{1}(t)+Q(0) f_{2}(t)+k_{o p} v f_{3}(t)\right. \\
& \left.+\int_{0}^{t} f_{1}(t-\tau) \theta(\tau) \mathrm{d} \tau\right\},
\end{aligned}
$$

where $f_{1}(t), f_{2}(t)$, and $f_{3}(t)$ are defined respectively as

$$
\begin{aligned}
& f_{1}(t)=\mathcal{L}^{-1}\left[\frac{1}{s\left[M\left(s^{2}+\frac{k_{o p}}{M}\right)+\xi(s) s\right]}\right] \\
& f_{2}(t)=\mathcal{L}^{-1}\left[\frac{[M s+\xi(s)]}{s\left[M\left(s^{2}+\frac{k_{o p}}{M}\right)+\xi(s) s\right]}\right] \\
& f_{3}(t)=\mathcal{L}^{-1}\left[\frac{1}{s^{3}\left[M\left(s^{2}+\frac{k_{o p}}{M}\right)+\xi(s) s\right]}\right] .
\end{aligned}
$$

In order to derive an analytical expression for the work distribution $P(W, t)$, we first define it as

$$
P(W, t)=\left\langle\delta\left(W-\int_{0}^{t} \frac{\partial H}{\partial \tau} \mathrm{d} \tau\right)\right\rangle,
$$

use the delta function expression,

$$
\delta(x)=\frac{1}{2 \pi} \int_{-\infty}^{+\infty} \exp (i k x) \mathrm{d} k
$$

and (15), to express $P(W, t)$ in an integral form as

$$
\begin{aligned}
& P(W, t)=\frac{1}{2 \pi A} \int_{-\infty}^{+\infty} \mathrm{d} k \int_{-\infty}^{+\infty} \mathrm{d} P \int_{-\infty}^{+\infty} \mathrm{d} Q \int_{-\infty}^{+\infty} \mathrm{d} \bar{p}_{1} \ldots \mathrm{d} \bar{p}_{N} \\
& \int_{-\infty}^{+\infty} \mathrm{d} \bar{q}_{1} \ldots \mathrm{d} \bar{q}_{N} \exp \left\{i k\left[W-\int_{0}^{t} \frac{\partial H}{\partial \tau} \mathrm{d} \tau\right]\right\} \exp \left[-\frac{H}{k_{B} T}\right],
\end{aligned}
$$

where, the normalization constant, $A$ is defined as

$$
A=\int_{-\infty}^{+\infty} \mathrm{d} P \int_{-\infty}^{+\infty} \mathrm{d} Q \int_{-\infty}^{+\infty} \mathrm{d} \bar{p}_{1} \ldots \mathrm{d} \bar{p}_{N} \int_{-\infty}^{+\infty} \mathrm{d} \bar{q}_{1} \ldots \mathrm{d} \bar{q}_{N}
$$




$$
\begin{aligned}
& \exp \left\{-\frac{1}{k_{B} T}\left[\frac{P^{2}}{2 M}+\frac{1}{2} k_{o p} Q^{2}\right.\right. \\
& \left.\left.+\sum_{j}\left(\frac{\bar{p}_{j}^{2}}{2 \bar{m}_{j}}+\frac{1}{2} \bar{m}_{j} \omega_{j}^{2}\left(\bar{q}_{j}-Q\right)^{2}\right)\right]\right\} .
\end{aligned}
$$

We now substitute the expression for the model Hamiltonian given by (6), the integral

$$
\int_{0}^{t} \frac{\partial H}{\partial \tau} \mathrm{d} \tau
$$

given by (15) and the random force $\theta(t)$ given by (12) into (18) to obtain

$$
\begin{aligned}
& P(W, t)=\frac{1}{2 \pi A} \int_{-\infty}^{+\infty} \mathrm{d} k \int_{-\infty}^{+\infty} \mathrm{d} P \int_{-\infty}^{+\infty} \mathrm{d} Q \\
& \int_{-\infty}^{+\infty} \mathrm{d} \bar{p}_{1} \ldots \mathrm{d} \bar{p}_{N} \int_{-\infty}^{+\infty} \mathrm{d} \bar{q}_{1} \ldots \mathrm{d} \bar{q}_{N} \\
& \exp \left\{i k\left[W-k_{o p} v^{2} t^{2} / 2-k_{o p}^{2} v^{2} f_{3}(t)\right]\right. \\
& +k k_{o p} v\left[P(0) f_{1}(t)+Q(0) f_{2}(t) \int_{0}^{t} f_{1}(t-\tau)\right. \\
& \sum_{j} \sqrt{\bar{m}_{j}} \omega_{j}\left[\sqrt{\bar{m}_{j}} \omega_{j}\left(\bar{q}_{j}-Q\right) \cos \omega_{j} \tau\right. \\
& \left.\left.\left.+\frac{\bar{p}_{j}}{\sqrt{\bar{m}_{j}}} \sin \omega_{j} \tau\right]\right]\right\} \\
& \exp \left\{-\frac{1}{k_{B} T}\left[\frac{P^{2}}{2 M}+\frac{1}{2} k_{o p} Q^{2}\right.\right. \\
& \left.\left.+\sum_{j}\left(\frac{\bar{p}_{j}^{2}}{2 \bar{m}_{j}}+\frac{1}{2} \bar{m}_{j} \omega_{j}^{2}\left(\bar{q}_{j}-Q\right)^{2}\right)\right]\right\},
\end{aligned}
$$

which, after some algebra, can be simplified as

$$
P(W, t)=\int_{-\infty}^{+\infty} \mathrm{d} k \exp \left\{-\left(1 / 2 k_{B} T\right) k^{2} k_{o p} v^{2}\right.
$$

$$
\begin{aligned}
& {\left[k_{o p} M f_{1}(t)^{2}+f_{2}(t)^{2}\right.} \\
& \left.+k_{o p} \int_{0}^{t} f_{1}\left(t-\tau_{1}\right) f_{1}\left(t-\tau_{2}\right) \xi\left(\tau_{1}-\tau_{2}\right) \mathrm{d} \tau_{2} \mathrm{~d} \tau_{2}\right] \\
& \left.+i k\left[W-k_{o p} v^{2} t^{2} / 2-k_{o p}^{2} v^{2} f_{3}(t)\right]\right\} .
\end{aligned}
$$

On evaluation of the Gaussian integral in this equation, we obtain the final simple expression

$$
P(W, t)=\frac{1}{\sqrt{2 \pi \sigma^{2}}} \exp \left(-(W-\langle W\rangle)^{2} / 2 \sigma^{2}\right),
$$

where $\langle W\rangle$ and $\sigma^{2}$ are given by

$$
\langle W\rangle=k_{o p} v^{2} t^{2} / 2-k_{o p}^{2} v^{2} f_{3}(t)
$$

and

$$
\begin{aligned}
\sigma^{2}= & 2 k_{B} T k_{o p} v^{2}\left[k_{o p} M f_{1}(t)^{2}+f_{2}(t)^{2}\right. \\
& \left.+k_{o p} \int_{0}^{t} \int_{0} f_{1}\left(t-\tau_{1}\right) f_{1}\left(t-\tau_{2}\right) \xi\left(\tau_{1}-\tau_{2}\right) \mathrm{d} \tau_{1} \mathrm{~d} \tau_{2}\right] .
\end{aligned}
$$

Equation (22) is the main new result of our work where the non-Markovian effect is explicitly incorporated in contrast to the results of Astumian ${ }^{9}$ where only the Markovian effect is considered.

The work distribution thus can be evaluated once the friction constant $\xi(t)$ is known for the solvent, which in turn depends on the mass $\left\{m_{j}\right\}$, frequencies $\left\{\omega_{j}\right\}$ and the coupling constant $\left\{c_{j}\right\}$ through (11).

For simplicity, we assume in (11) all the masses as well as the coupling constants to be equal, i.e. $m_{j}=m$, and $c_{j}=(C / \sqrt{N})$ for all values of $j$. To replace the sum by an integral, we assume that there is an infinitely large number of oscillators in the heat bath, with a continuous distribution of frequencies, and use the replacement

$$
\sum_{j} \rightarrow \sum_{\omega_{j}} \rightarrow N \int_{0}^{\infty} g(\omega) \mathrm{d} \omega
$$

where $g(\omega)$ is the density of states. Under these assumptions, (11) can be written as 


$$
\xi(t)=\frac{C^{2}}{m} \int_{0}^{\infty} \mathrm{d} \omega \frac{g(\omega)}{\omega^{2}} \cos (\omega t)
$$

Now considering the density of states, as considered by Zwanzig, ${ }^{11}$ to be quadratic in $\omega$ below a cut-off frequency $\omega_{c}$ and have the form

$$
\begin{aligned}
g(\omega) & =\frac{3 \omega^{2}}{\omega_{c}^{3}}, \omega<\omega_{c}, \\
& =0, \omega>\omega_{c},
\end{aligned}
$$

we finally obtain the expression

$$
\xi(t)=\xi(0) \frac{\sin \left(\omega_{c} t\right)}{\omega_{c} t}
$$

where

$$
\xi(0) \frac{3 C^{2}}{m \omega_{c}^{2}}
$$

Now taking the Laplace transform on both sides of (28) we obtain

$$
\xi(s)=\frac{\xi(0)}{\omega_{c}} \tan ^{-1}\left(\frac{\omega_{c}}{s}\right) .
$$

In the optical trap experiment of Wang et $a l^{4}$, the important question is that what is the dissipative part of the total work $\langle W\rangle$ which is responsible for the heat or entropy production in the system as a result of the friction of the particle with the surrounding fluid. One can show ${ }^{17}$ that the total work is the sum of the mechanical work and the dissipative work, i.e.

$$
\langle W\rangle=W_{\text {mech }}+W_{\text {diss }}
$$

where

$$
W_{\text {mech }}=\frac{k_{o p}}{2}\left\langle\left\{Q(t)-Q^{*}\right\}^{2}-Q(0)^{2}\right\rangle,
$$

and

$$
W_{\text {diss }}=\left\langle\int_{0}^{t} \dot{Q}(\tau) F_{o p}(\tau) \mathrm{d} \tau\right\rangle,
$$

where $Q^{*}=v t$, denotes the instantaneous coordinate of the optical trap. The average of the square of fluctuation of the colloidal particle coordinate $Q(t)$ with respect to the optical centre $Q^{*}$ is defined as

$$
\left\langle\left\{Q(t)-Q^{*}\right\}^{2}\right\rangle=\int_{-\infty}^{+\infty} \gamma^{2} P(\gamma, t) \mathrm{d} \gamma,
$$

where

$$
P(\gamma, t)=\left\langle\delta\left(\left\{Q(t)-Q^{*}\right\}-\gamma\right)\right\rangle .
$$

Now substituting the expression of $Q(t)\left(=\mathcal{L}^{-1} \tilde{Q}(s)\right)$ in (34), we obtain

$$
P(\gamma, t)=\sqrt{\frac{\beta}{2 \pi G(t)}} \exp \left[-\frac{\beta}{2 G(t)}\left\{\gamma+\left(Q^{*}-k_{o p} v g_{3}(t)\right)\right\}^{2}\right],
$$

where

$$
\begin{aligned}
G(t)= & M g_{1}^{2}(t)+\frac{g_{2}^{2}(t)}{k_{o p}} \\
& +\int_{0}^{t} \int_{0}^{t} g_{1}\left(t-\tau_{1}\right) g_{1}\left(t-\tau_{2}\right) \xi\left(\tau_{1}-\tau_{2}\right) \mathrm{d} \tau_{1} \mathrm{~d} \tau_{2} .
\end{aligned}
$$

and

$$
\begin{aligned}
& g_{1}(t)=\mathcal{L}^{-1}\left(\frac{1}{\left[M\left(s^{2}+\frac{k_{o p}}{M}\right)+\xi(s) s\right]}\right) \\
& g_{2}(t)=\mathcal{L}^{-1}\left(\frac{[M s+\xi(s)]}{\left[M\left(s^{2}+\frac{k_{o p}}{M}\right)+\xi(s) s\right]}\right) \\
& g_{3}(t)=\mathcal{L}^{-1}\left(\frac{1}{s^{2}\left[M\left(s^{2}+\frac{k_{o p}}{M}\right)+\xi(s) s\right]}\right] .
\end{aligned}
$$

Now substituting (35) in (33), we obtain

$$
\frac{k_{o p}}{2}\left\langle\left\{Q(t)-Q^{*}\right\}^{2}\right\rangle=\frac{k_{o p}}{2}\left[k_{B} T G(t)+K^{2}(t)\right],
$$

where

$$
K(t)=Q^{*}-k_{o p} v g_{3}(t) .
$$


The average potential energy of the colloidal particle at equilibrium is defined as

$$
\left(k_{o p} / 2\right)\left\langle Q(0)^{2}\right\rangle=1 / 2 \beta
$$

Now combining (30), (40) and (42), we obtain

$$
\frac{W_{\mathrm{diss}}}{\langle W\rangle}=1+\frac{1}{2 \beta\langle W\rangle}\left[1-k_{o p} G(t)\right]-\frac{k_{o p}}{2\langle W\rangle} K^{2}(t)
$$

Equation (43) is another new result of our work representing the fraction of the total work done on the colloidal particle that is converted into heat which is responsible for the production of entropy.

\section{Results and discussion}

It is clear from (16), (22)-(24) and (29) that $P(W, t)$ depends explicitly on the parameters such as the optical strength $k_{o p}$, cut-off frequency $\omega_{c}$, mass $(M)$ of the colloidal particle, pulling speed $v$ etc. Instead of using the actual values of these parameters, it is convenient to express $P(W, t)$ in terms of the dimensionless quantities viz. $t^{*}\left(=\omega_{c} t\right), E^{*}\left(=M v^{2} / k_{B} T\right)$, $k_{o p}^{*}\left(=k_{\mathrm{op}} / \xi(0)\right), \omega^{*}\left(=\left(k_{o p} / M \omega_{\mathrm{c}}^{2}\right)^{1 / 2}\right)$, etc. We first substitute (29) into (16a), (16b) and (16c) and then carry out the inverse Laplace transform numerically to obtain $f_{1}(t), f_{2}(t)$ and $f_{3}(t)$. The work distribution $P(W, t)$ is then calculated at different values of time and plotted in figure 1 for the parameters $E^{*}=1 \cdot 0$, $k_{o p}^{*}=0.5$ and $\omega^{*}=1.0$. It is clear from the figure

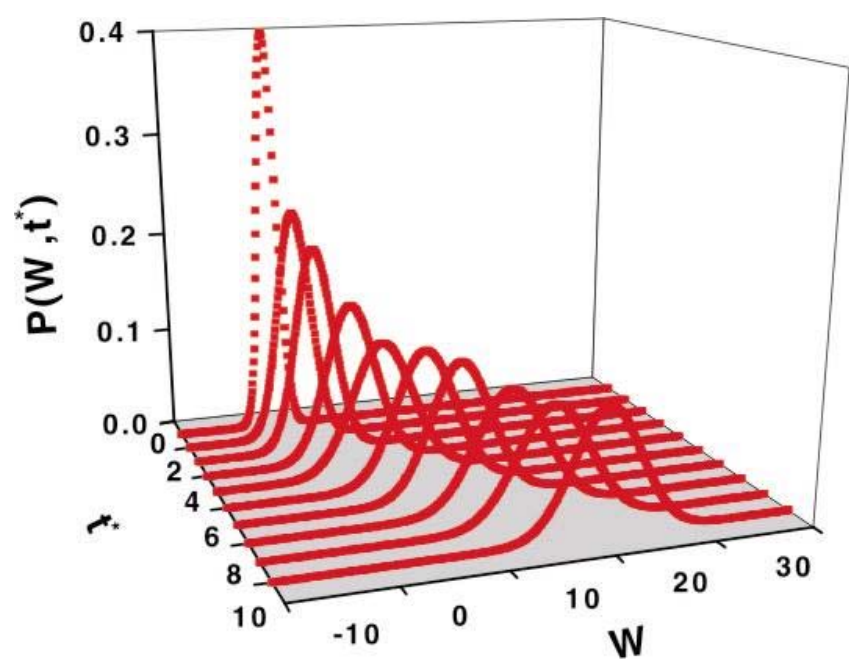

Figure 1. 3D-plot for the work distribution with respect to time $\left(t^{*}\right)$ at constant velocity of dragging for the parameter values $\omega^{*}=1 \cdot 0, k_{o p}^{*}=0.5$ and $E^{*}=1 \cdot 0$. that when the pulling time is small, the work distribution is sharply peaked whereas with increasing pulling time, the work distribution becomes broader and the peak is shifted towards higher value of $W$. For fixed values of the physical parameters, the average work $\langle W\rangle$ done on the system increases with an increase of pulling time, as a result of which the peak for the work distribution $P(W, t)$ is expected to shift towards higher value of $W$, since the major contribution to the average

$$
\langle W\rangle\left(=\int_{-\infty}^{\infty} W P(W, t) \mathrm{d} W\right)
$$

is contributed from the peak value of $P(W, t)$.

Next we consider the case when both the pulling speed $v$ and time $t$ are varied but they are adjusted in such a way that the distance travelled by the optical centre is constant $\left(E^{*} . t^{*^{2}}=\right.$ constant). We have calculated $P(W, t)$ under this constraint, for the parameter values $k_{o p}^{*}=0.5$ and $\omega^{*}=1.0$ and plotted the calculated results in figure 2. It is clear from the figure that when the pulling speed is small but pulling time is large, the distribution function $P(W, t)$ is sharply peaked, whereas when the pulling speed is high but pulling time is small, $P(W, t)$ is broadened. When the pulling speed is very small, one has the case of adiabatic limit and the system passes from the initial state to the final state through the quasiequilibrium states which leads to the work distribution sharply peaked. On the other hand, when the pulling speed is high but pulling time is small, one

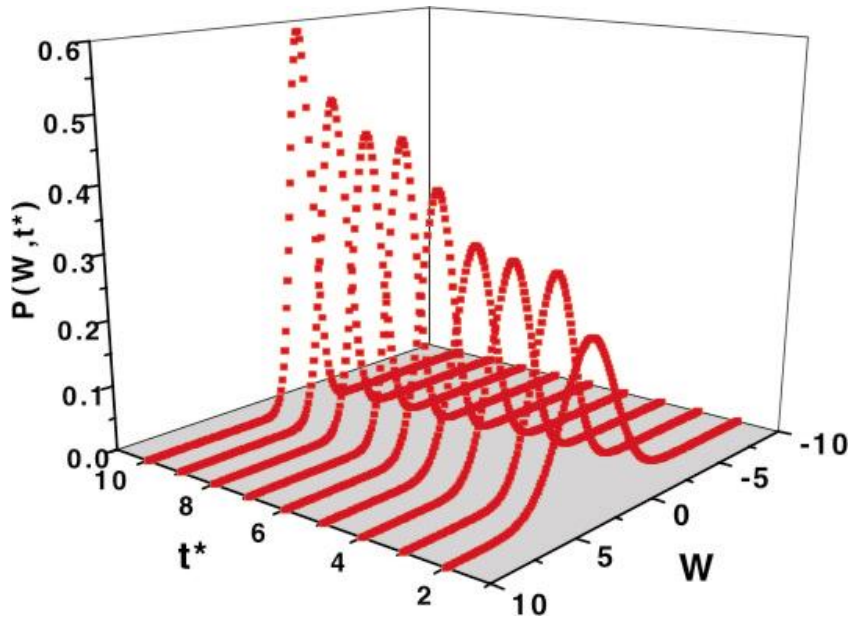

Figure 2. 3D-plot for the work distribution with respect to time $\left(t^{*}\right)$ at constant displacement for the parameter values $E^{*} \cdot t^{*^{2}}=1 \cdot 0, \omega^{*}=1 \cdot 0$ and $k_{o p}^{*}=0.5$. 
has a non-equilibrium situation and the work distribution is expected to be broad. In order to study the effect of pulling speed of the colloidal particle on the probability distribution $P(W, t)$ for a given pulling time we consider three different cases, viz. $E^{*}=1 \cdot 0,2.0$ and 3.0 and the calculated values for the parameters $k_{o p}^{*}=0.5$ and $\omega^{*}=1.0$ and fixed time $t^{*}=3 \cdot 0$, are plotted in figure 3 . It is clear from the figure that the probability of doing more work on the colloidal particle is more probable for higher pulling speed. With increase of the pulling speed, the average work $\langle W\rangle$ done on the colloidal particle

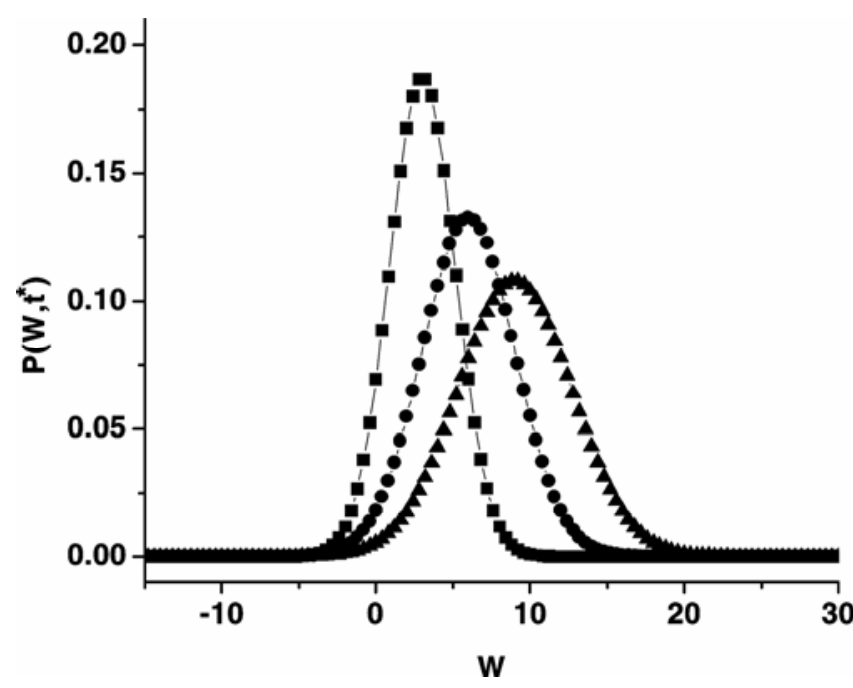

Figure 3. Variation of the work distribution for different values of dragging velocities $(v)$ for the parameter values $\omega^{*}=1 \cdot 0, k_{o p}^{*}=0 \cdot 5$ and $t^{*}=3 \cdot 0$. The '--n--' line, '--•--'line and '-- $\Delta--$ 'line corresponds to $E^{*}=1 \cdot 0,2 \cdot 0$ and $3 \cdot 0$ respectively.

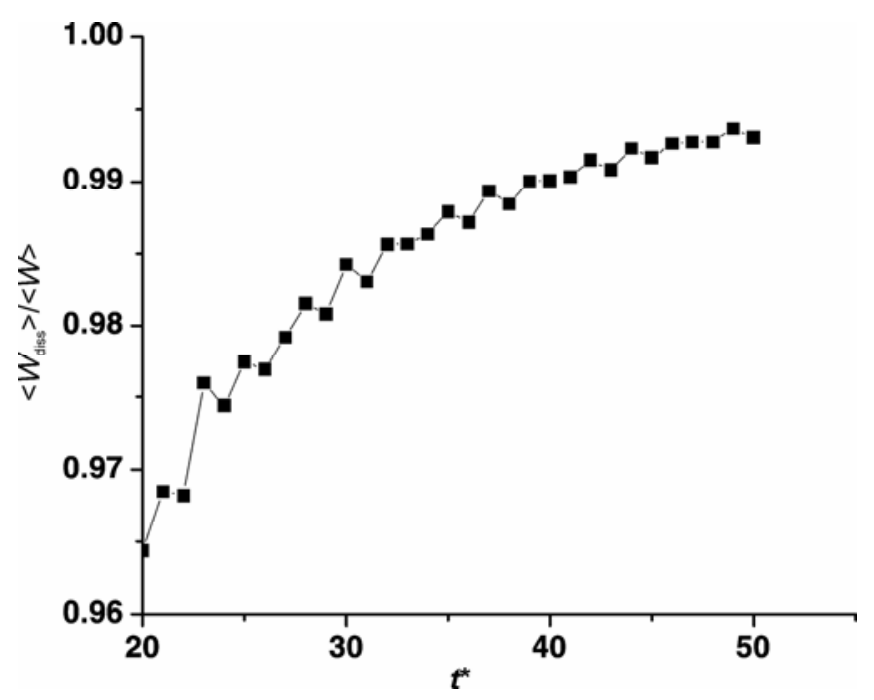

Figure 4. Variation of ratio of the average dissipation work to the average of total microscopic work for the parameter values $\omega^{*}=1 \cdot 0, k_{o p}^{*}=0 \cdot 5$ and $E^{*}=2 \cdot 0$. increases leading to broadening of the distribution function and a shift of the peak value of the distribution function $P(W, t)$ to a more positive value of $W$. Another important quantity is $W_{\text {diss }} \mid\langle W\rangle$ which is a measure of the fraction of the total work $\langle W\rangle$ that goes as dissipation or entropy production due to the friction of the solute particle with the solvent molecules. We have calculated this quantity for the parameters $k_{o p}^{*}=0.5, \omega^{*}=1.0$ and $E^{*}=2.0$ and plotted the results in figure 4 . It is clear from the figure that only at long time, the total work is completely converted into dissipation or entropy production.

\section{Conclusions}

We have proposed here a simple approach to derive an exact analytical expression for the work distribution for a system consisting of a colloidal particle trapped in an optical harmonic potential well and the centre of the well is being pulled with constant velocity through a solution represented by non-Markovian bath in contrast to the earlier studies, ${ }^{11}$ where only Markovian bath has been considered. The thermal bath is assumed here to be composed of an infinite set of harmonic oscillators and the model Hamiltonian is constructed by considering linear dissipative mechanism for their coupling with the trapped colloidal particle. We have derived an analytical expression for the distribution of work and study the effects of pulling time, pulling speed, adiabatic limit and fraction of conversion of total work into the dissipative form. While this work was under review, we came to know of a preprint by Chakrabarti ${ }^{18}$ who has studied a similar model system to verify the transient state work fluctuation theorem. However, the objective of the present work as well as the quantities investigated here are different. Although we have considered here a constant speed of the optical centre, it will be interesting to consider the case when the speed of optical centre varies arbitrarily. Work in this direction is in progress.

\section{Acknowledgements}

We thank the anonymous referee for bringing Ref. 18 to our attention.

\section{References}

1. (a) Jarzynski C 1997 Phys. Rev. Lett. 78 2690; (b) Jarzynski C 1997 Phys. Rev. E56 5018 
2. Liphardt J, Dumont S, Smith S B, Tinoco Jr I and Bustamante C 2002 Science 2961832

3. Collin D, Ritort F, Jarzynski C, Smith S, Tinoco I and Bustamante C 2005 Nature (London) 437231

4. Wang G M, Sevick E M, Mittag E, Searles D J and Evans D J 2002 Phys. Rev. Lett. 89050601

5. Carberry D M, Reid J C, Wang G M, Sevick E M, Searles D J and Evans D J 2004 Phys. Rev. Lett. 92 140601

6. Trepagnier E H, Jarzynski C, Ritort F, Crooks G E, Bustamante C J and Liphardt J 2004 Proc. Natl. Acad. Sci. USA 10115038

7. Blickle V, Speck T, Helden L, Seifert U and Bechinger C 2006 Phys. Rev. Lett. 96070603

8. Narayan O and Dhar A 2004 J. Phys. A: Math. Gen. 3763
9. Mai T and Dhar A 2007 Phys. Rev. E75 061101

10. Mazonka O and Jarzynski C 1999 arXiv.cond-mat/ 9912121

11. Astumian R D 2007 J. Chem. Phys. 126111102

12. Zwanzig R J 1973 Stat. Phys. 9215

13. Pollak E 1986 Chem. Phys. Lett. 127178

14. Samanta A and Ghosh S K 2008 J. Phys. Chem. $\mathbf{A 1 1 2}$ 752

15. Mukamel S 1995 Principles of nonlinear optical spectroscopy (New York: Oxford University Press)

16. Dattagupta S and Singh J 1997 Phys. Rev. Lett. 79 961

17. van Zon R and Cohen E G D 2003 Phys. Rev. E67 046102

18. Chakrabarti R 2009 Pramana-J. Phys. 72665 